University of Nebraska - Lincoln

DigitalCommons@University of Nebraska - Lincoln

6-1-1999

\title{
Self-focusing, channel formation, and high-energy ion generation in interaction of an intense short laser pulse with a He jet
}

\author{
G.S. Sarkisov \\ P. N. Lebedev Physics Institute, Russian Academy of Science, Moscow, Russia \\ B. Yu. Bychenkov \\ P. N. Lebedev Physics Institute, Russian Academy of Science, Moscow, Russia \\ V.N. Novikov \\ P. N. Lebedev Physics Institute, Russian Academy of Science, Moscow, Russia \\ V.T. Tikhonchuk \\ P. N. Lebedev Physics Institute, Russian Academy of Science, Moscow, Russia \\ Anatoly Maksimchuk \\ University of Michigan, tolya@umich.edu \\ See next page for additional authors
}

Follow this and additional works at: https://digitalcommons.unl.edu/physicsumstadter

Part of the Physics Commons

Sarkisov, G.S.; Bychenkov, B. Yu.; Novikov, V.N.; Tikhonchuk, V.T.; Maksimchuk, Anatoly; Chen, Shouyuan; Wagner, R.; Mourou, G.; and Umstadter, Donald, "Self-focusing, channel formation, and high-energy ion generation in interaction of an intense short laser pulse with a He jet" (1999). Donald Umstadter Publications. 7.

https://digitalcommons.unl.edu/physicsumstadter/7

This Article is brought to you for free and open access by the Research Papers in Physics and Astronomy at DigitalCommons@University of Nebraska - Lincoln. It has been accepted for inclusion in Donald Umstadter Publications by an authorized administrator of DigitalCommons@University of Nebraska - Lincoln. 


\section{Authors}

G.S. Sarkisov, B. Yu. Bychenkov, V.N. Novikov, V.T. Tikhonchuk, Anatoly Maksimchuk, Shouyuan Chen, R. Wagner, G. Mourou, and Donald Umstadter 


\title{
Self-focusing, channel formation, and high-energy ion generation in interaction of an intense short laser pulse with a He jet
}

\author{
G. S. Sarkisov, * V. Yu. Bychenkov, V. N. Novikov, and V. T. Tikhonchuk \\ P. N. Lebedev Physics Institute, Russian Academy of Science, Moscow 117924, Russia \\ A. Maksimchuk, S.-Y. Chen, R. Wagner, G. Mourou, and D. Umstadter \\ Center for Ultrafast Optical Science, University of Michigan, Ann Arbor, Michigan 48109-2099
}

(Received 28 September 1998)

\begin{abstract}
Using interferometry, we investigate the dynamics of interaction of a relativistically intense 4-TW, 400-fs laser pulse with a He gas jet. We observe a stable plasma channel $1 \mathrm{~mm}$ long and less than $30 \mu \mathrm{m}$ in diameter, with a radial gradient of electron density $\sim 5 \times 10^{22} \mathrm{~cm}^{-4}$ and with an on-axis electron density approximately ten times less than its maximum value of $8 \times 10^{19} \mathrm{~cm}^{-3}$. A high radial velocity of the surrounding gas ionization of $\sim 3.8 \times 10^{8} \mathrm{~cm} / \mathrm{s}$ has been observed after the channel formation, and it is attributed to the fast ions expelled from the laser channel and propagating radially outward. We developed a kinetic model which describes the plasma channel formation and the subsequent ambient gas excitation and ionization. Comparing the model predictions with the interferometric data, we reconstructed the axial profile of laser channel and on-axis laser intensity. The estimated maximum energy of accelerated ions is about $500 \mathrm{keV}$, and the total energy of the fast ions is 5\% of the laser pulse energy. [S1063-651X(99)07505-4]
\end{abstract}

PACS number(s): 52.40.Nk, 52.75.Di, 52.35.Mw

\section{INTRODUCTION}

A number of proposed applications of ultrahigh intensity short laser pulses require laser guiding for distances much longer than a Rayleigh length without considerable energy loss and significant diffraction. A stable, long-lived channel in a plasma can serve as an optical waveguide for the fast ignitor [1] and laser wake-field particle acceleration [2,3] experiments. Guiding of intense laser pulses in underdense plasmas at relativistic intensities was first reported in Ref. [4], and then studied more completely in Refs. [5-10]. The initial phase of plasma channeling is governed by selffocusing due to electron expulsion by the laser ponderomotive force and the relativistic increase of the electron mass. Both these effects increase the refractive index of a plasma on axis and further focus the laser pulse. A significant part of the laser pulse can be trapped, form a laser channel, and propagate many Rayleigh lengths without divergence [1114].

The effects of terawatt laser beam self-focusing and plasma channel formation have recently been observed using the laser probing technique. The shadowgraphy study of the onset of relativistic self-focusing using 0.3-TW, 250-fs Ti:sapphire laser pulse with an intensity $\sim 10^{17} \mathrm{~W} / \mathrm{cm}^{2}$ in underdense gas jet plasmas was presented in Ref. [15]. A plasma channel expansion with high velocity of $\sim 5 \times 10^{8}$ $\mathrm{cm} / \mathrm{s}$ was observed with the interferometric technique in Ref. [16] for the interaction of a $600-\mathrm{fs}, 5 \times 10^{18} \mathrm{~W} / \mathrm{cm}^{2}$ laser pulse with a solid target. Interferometric observations of high temperature (above $1 \mathrm{keV}$ ) plasma filaments in the interaction of a 2-ps laser pulse with an intensity $\sim 10^{16} \mathrm{~W} / \mathrm{cm}^{2}$

\footnotetext{
*Present address: Department of Physics, University of Nevada, Reno, NV 89557-0058.
}

with atomic-cluster gases was reported by Ditmire et al. [17]. Observations of terawatt-laser pulse propagation inside a $200-\mu \mathrm{m}$-diameter plasma channel in neon and helium gas jets using the Moire technique were presented in Ref. [18].

The diagnostic methods used in Refs. [15-18] do not resolve subpicosecond time scales, and therefore cannot be used to observe laser pulse self-focusing related to plasma motion during the laser pulse. Although the self-focusing of a short laser pulse is an electron-dominated process, on a picosecond time scale ions also become involved, and selfconsistent ion and electron motion results in a deeper plasma channel with larger diameter in a later time. The effect of ion motion induced by a short relativistic laser pulse was studied in hydrodynamic simulations [19] for a 100-ps time scale. However, the hydrodynamic approach is not relevant for ions at the relativistic laser intensities, since the mean free path of the accelerated ions could be much larger than the channel diameter. Full electromagnetic three-dimensional particle-incell (PIC) simulations [20,21] could be appropriate for such conditions; however, these PIC simulations are limited to a time scale of a few picoseconds even with current high performance computers.

This paper presents experimental results and their theoretical interpretation on the dynamics of a plasma channel produced by a high intensity short laser pulse propagating in a plasma created by the ionization of an initially neutral $\mathrm{He}$ gas jet. Relativistic self-focusing and self-channeling of the laser pulse and the dynamics of ambient gas excitation and ionization were observed by interferometric and shadow techniques. Some results on the channel formation and plasma density dynamics have been published recently elsewhere $[22,23]$. The experiment is modeled with a simple cold ion kinetic code, which includes the electrostatic field governed by the laser driven relativistic ponderomotive force in a cylindrical geometry. It also accounts for the inelastic collisions of fast ions and accompanying electrons with the ambient gas, and ignores thermal pressure effects. A com- 
parison of model results with the observations allows us to reconstruct the characteristics of the self-focused laser beam and the laser-produced plasma channel.

The paper is organized as follows. In Sec. II we describe the experimental setup, and present experimental evidence for channel formation and results on channel dynamics. In Sec. III a simple kinetic model for plasma cavitation and high-energy ion generation is formulated. An interpretation of the experimental results is presented in Sec. IV. It is demonstrated that the kinetic model quantitatively reproduces the observed phenomena and provides two important conclusions: (i) the evolution of the plasma density profile dramatically disagrees with the hydrodynamic calculations, reported in Ref. [19], and (ii) excited He atoms make a significant contribution to the plasma refraction index. A discussion, several predictions of ion acceleration for more intense laser beams, and a summary are presented in Sec. V.

\section{INTERFEROMETRIC INVESTIGATIONS OF A CHANNEL FORMATION}

\section{A. Experimental setup}

The experiment was performed using a 10-TW Ti:sapphire-Nd:glass laser system based on chirped-pulse amplification [24] developed at the Center for Ultrafast Optical Science, University of Michigan. The laser operates at a wavelength of $\lambda_{0}=1.053 \mu \mathrm{m}$, and produces pulses with an energy up to $4 \mathrm{~J}$ in $400 \mathrm{fs}$ [full width at half maximum $((\mathrm{FWHM})]$ with an intensity contrast ratio $\sim 10^{5}: 1$, as measured by the third order correlation technique [25]. The 50mm-diameter laser beam was focused with an off-axis parabolic mirror $(f / 3.3, f=16.5 \mathrm{~cm})$ to a $10-\mu \mathrm{m}$ spot with a vacuum intensity of $6 \times 10^{18} \mathrm{~W} / \mathrm{cm}^{2}$. The radial distribution of the laser intensity also demonstrates $\sim 50$ - $\mu \mathrm{m}$-diameter wings that contain approximately $10 \%$ of the laser energy. The laser beam was focused in a high backing pressure $(\sim 7$ $\mathrm{MPa}) \mathrm{He}$ gas jet expanding through a 1-mm-diameter nozzle [Fig. 1(a)]. The optimal conditions for beam guiding correspond to the laser focusing on the jet edge at a distance $x$ $=0.5-0.75 \mathrm{~mm}$ from the nozzle, where the He atom density is $\sim 4 \times 10^{19} \mathrm{~cm}^{-3}$.

The layout of two-channel optical system for simultaneous recording of interferometric and shadow plasma images is shown in Fig. 1(b). The diagnostic beam (2) with a wavelength of $\lambda=1.053 \mu \mathrm{m}$ was obtained by splitting $\sim 5 \%$ of the pump pulse, propagated through an adjustable motorized optical delay (5), and probed a plasma (7) in the direction perpendicular to the interaction beam. The plasma was imaged by spherical lens (8) with an angular aperture $7^{\circ}$ on two 12-bit CCD cameras (10-shadow) and (11interferogram). The spatial and temporal resolutions were 10 $\mu \mathrm{m}$ and $400 \mathrm{fs}$, respectively. The air-wedge shearing interferometer (9) was used for the electron density measurements. The principle of operation of this interferometer was described in Ref. [26].

\section{B. Experimental observations}

We followed the plasma evolution for a time period from -2 to $+55 \mathrm{ps}$. The time $t=0$ is taken to be the arrival time of the laser pulse peak to the focal plane $z=0$. We observed (a)
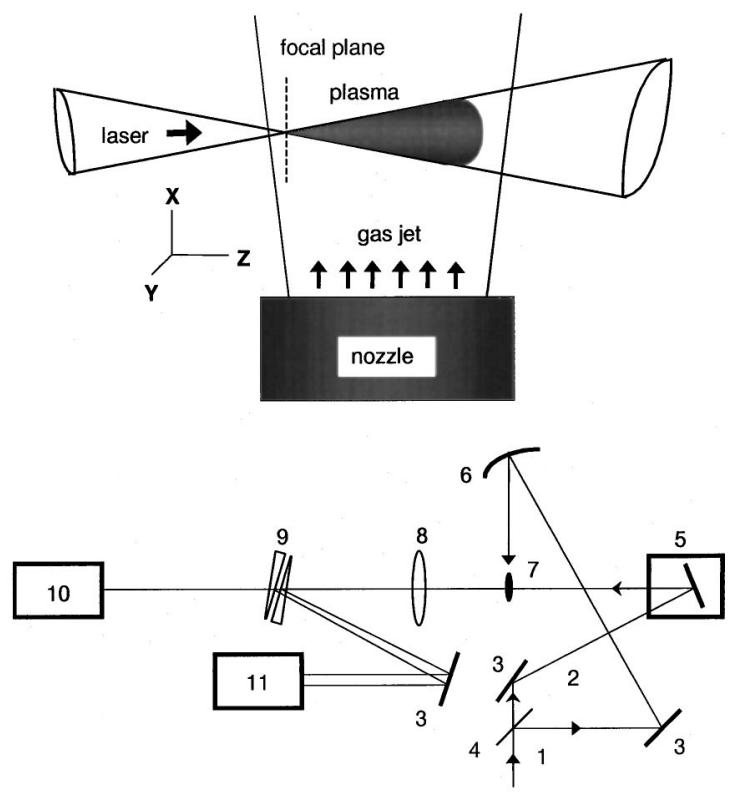

FIG. 1. A scheme of the laser-gas jet interaction (a) and an experimental setup (b): 1 , interaction beam; 2 , probe beam; 3 , mirrors; $4,5 \%$ beam splitter; 5 , motorized optical delay; 6 , off-axis parabolic mirror; 7, plasma from a gas jet; 8, spherical imaging lens; 9, air-wedge shearing interferometer; 10, shadow recording CCD; 11, interferogram recording CCD.

a fast gas ionization in the cone angle $\left(\sim 17^{\circ}\right)$ of the laser $\sim 2$ ps before the pump pulse arrival. The laser intensity in the temporal foot and in the spatial wings of the pulse was above the tunneling ionization threshold for He atoms, which is $\sim 10^{15} \mathrm{~W} / \mathrm{cm}^{2}$ to produce a single ionized ion $[15,27,28]$. The ionization front propagated along the laser axis with a subluminal velocity until it reached the rear side of the jet. The transverse size of the formed plasma was about $100 \mu \mathrm{m}$ in diameter, which is much greater than the laser focal spot. For the optical self-guiding of the laser pulses in the plasma, the radial profile of the index of refraction must have a maximum on axis, causing the wave front to curve inward and the laser beam to converge. When this focusing force is strong enough to counteract the diffraction of the beam, the laser pulse can propagate over a long distance while maintaining a small cross section (laser channel). For a Gaussian beam with a power exceeding a critical power $P_{c}=17 n_{c} / n_{e} G W$ (where $n_{c}$ is the critical density for the interaction laser pulse) self-focusing and self-channeling can occur as a result of two effects: (i) the relativistic increase of electron mass in

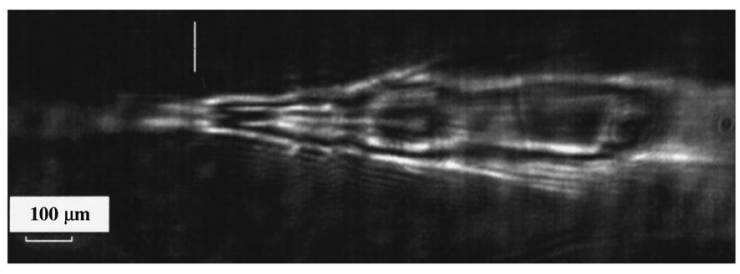

FIG. 2. A schlieren image of a plasma for the defocusing dominated regime. The plasma was probed at a 10-ps delay after the passage of the interaction pulse. The vertical line indicates the position of focal plane in a vacuum. 


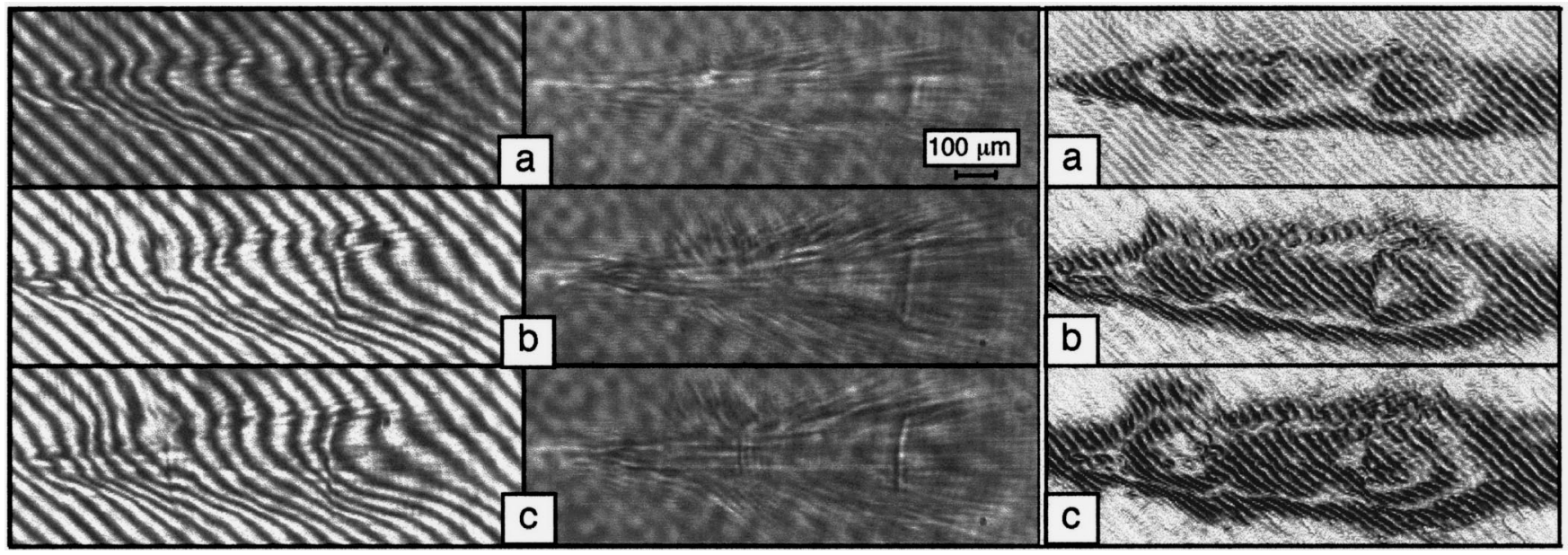

FIG. 3. Interferograms (left column), shadowgrams (center column), and topology of the wave front disturbance (right column) of a plasma in the defocusing dominated regime at different time delays: 5 ps (a), 10 ps (b), and 20 ps (c). The internal pulsation structure of the wave front isoline emphasizes an interplay between self-focusing and defocusing.

the laser field, and (ii) the reduction of the electron density on axis due to the expulsion of the electrons by the laser pondermotive force.

At first, we will describe an observation of the phenomena related to the regime of the onset of relativistic self-focusing. This regime is characterized by the formation of a short onaxis channel. Nevertheless, the main part of the laser energy has not been trapped in the channel, but has propagated inside the laser ionization cone, leading to a plasma density pulsation and off-axial laser filamentation. The laser beam had a power of less than $3 \mathrm{TW}$, and had been focused at a distance $x \sim 1.5 \mathrm{~mm}$ from the nozzle edge. We believe that even had the laser power exceeded the critical power by a few times, the beam diffraction would dominate in this regime. A typical schlieren image of plasma at time delay $t$ $=10 \mathrm{ps}$ after the interaction pulse is presented in Fig. 2. To produce a schlieren image, we horizontally placed a 400$\mu \mathrm{m}$-diameter wire in the focal plane of the imaging lens (8) [Fig. 1(b)]. Bright parts of the image correspond to the regions where the refraction index has high transversal gradients. There are two main features in Fig. 2: a bright, short, and narrow line on the laser axis, and nonuniform axial pulsations of the external cone of plasma. The first feature is due to channeling of a small part of the laser's energy. The

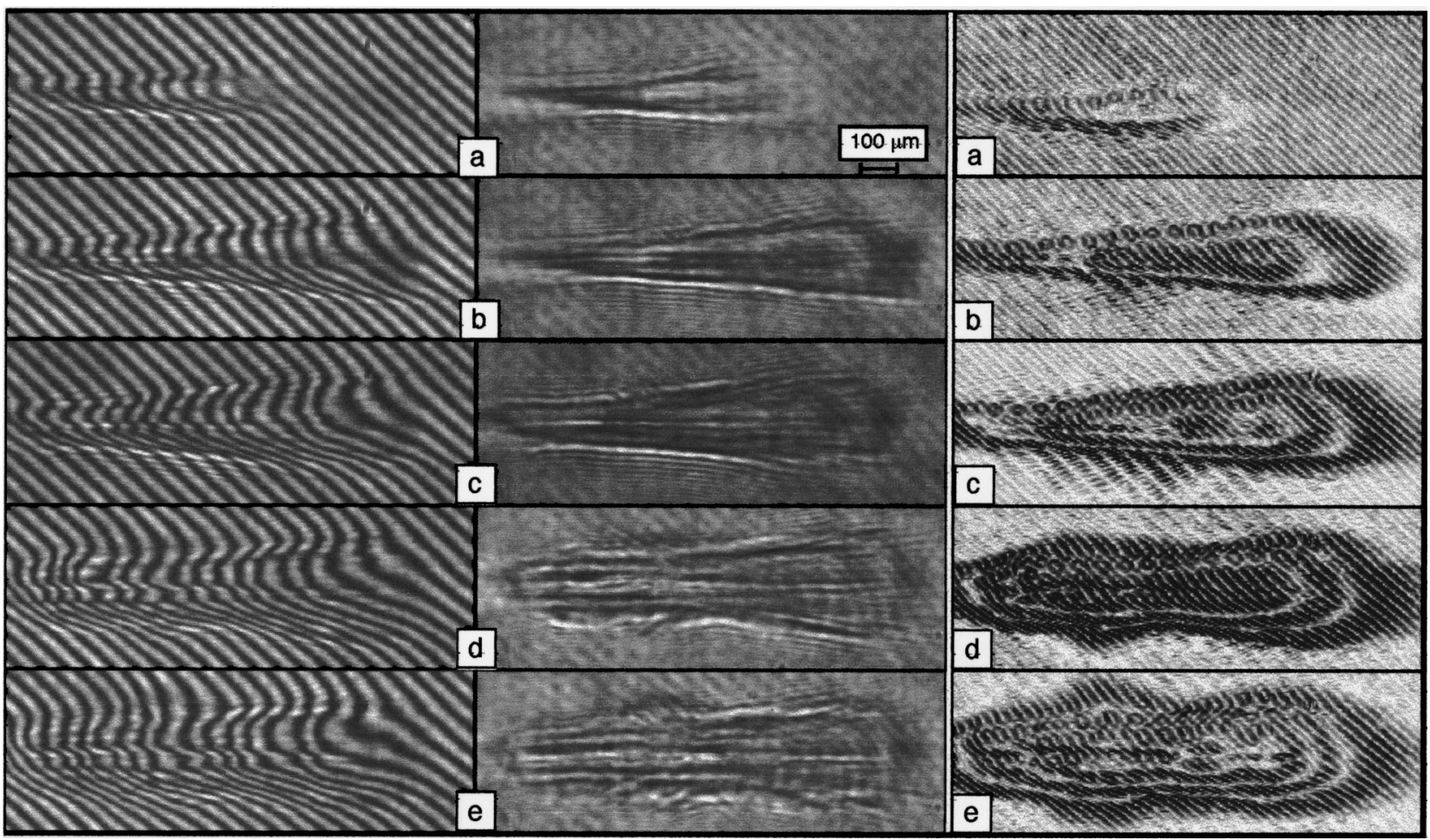

FIG. 4. Interferograms (left column), shadowgrams (central column), and topology of the wave front disturbance (right column) of a plasma in the regime of relativistic self-focusing at three different delays: 0 ps (a), 5 ps (b), 15 ps (c), 35 ps (d), and 45 ps (e). Contrary to Fig. 3, the internal pulsation structure of the wave front isoline is absent for the relativistic self-focusing regime. 


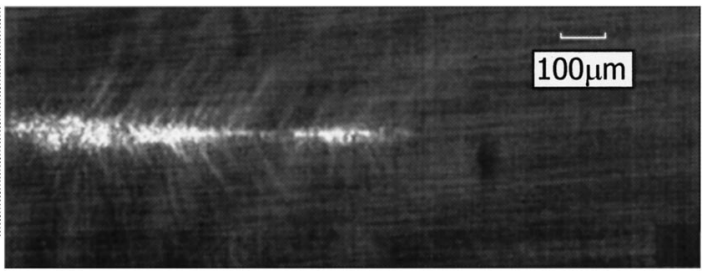

FIG. 5. Thomson scattering image of laser-He jet interaction at a wavelength of $1.053 \mu \mathrm{m}$ and a laser power of $4.3 \mathrm{TW}$. The direction of the main beam propagation is from the left to the right.

length of the axial channel is short, $\sim 300 \mu \mathrm{m}$, in this regime. The second feature is probably due to the interplay between ionization defocusing and the self-focusing of the pump beam. The temporal evolution of the plasma can be seen from the interferograms (left column) and shadow images (center column) in Fig. 3 at three subsequent time delays 5, 10, and $20 \mathrm{ps}$. For the identification of the wave front disturbance topology of the probe laser beam, we applied a special procedure to the interferogram processing by conversion from a noncontour type interferogram to a contour type interferogram. For that we subtracted the nondisturbed interferogram from the plasma interferogram, and then applied a "find edge" filter. For the image processing we used the public domain program SCION IMAGE [29]. The results of this procedure are presented in a right column of Fig. 3. The first outside black contour line corresponds to the $\lambda / 2$ phase shift, the next white contour line corresponds to the $\lambda$ phase shift, and so on. The isophase line of axial pulsations of the ionization cone are similar to those seen in the schlieren image in Fig. 2. The plasma structure in this regime is significantly different from low-energy experiments reported in Ref. [15], where only the laser filamentation in underdense plasma has been observed.

Second, we studied the regime of relativistic selffocusing. By optimal focusing of the laser beam at a distance $x=0.5 \mathrm{~mm}$ from the nozzle edge, we observed a long narrow channel on the laser axis. A significant part of the laser energy $(\sim 60 \%)$ is trapped in this narrow channel. To achieve the laser pulse trapping it was essential to have a high gas

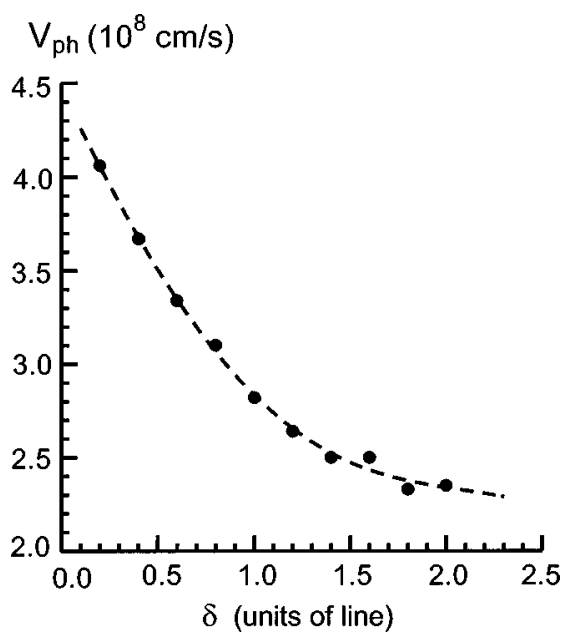

FIG. 6. Radial expansion velocity $V_{\mathrm{ph}}$ of the interference phase shift for the cross section $z=100 \mu \mathrm{m}$. One line corresponds to one wavelength phase shift.

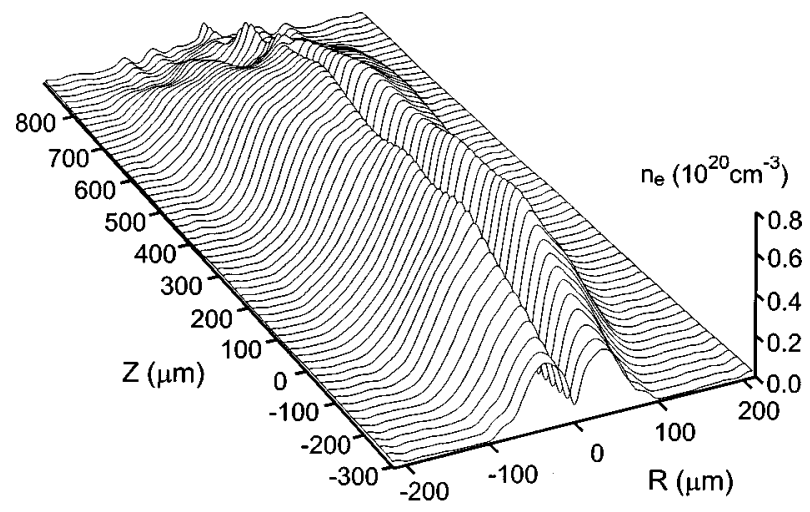

FIG. 7. Spatial distribution of the electron density for a time moment of 35 ps.

density gradient in the edge of the jet, and to focus the laser beam at the front edge of the jet. A typical set of interferograms (left column) and shadowgrams (center column) of a plasma, taken from the similar shots (laser power of $4.3 \mathrm{TW}$ ) at five subsequent time delays is presented in Fig. 4. The first signature of plasma channel formation was observed at $t$ $=0$ ps. The local opposite displacement of the interference fringes in a narrow axial region in the interferogram indicates a decrease of the phase shift, and hence a decrease of the on-axis electron density. This region is manifested in the shadow image by a bright long and narrow line. The channel length was about $1000 \mu \mathrm{m}$, and comparable to the gas jet length. One can see a significant difference of these plasma images from those of the previous regime of interaction (Figs. 2 and 3). We did not observe axial pulsations of the wave front isolines (right column of Fig. 4) or beam filamentation on shadowgrams, as in they other regime. This is because the main part of the laser energy now propagates inside the plasma channel, and the diffracted electromagnetic wave does not have enough power for the generation of the above mentioned structures.

In order to diagnose the spatial extent of the laser channel, we used $90^{\circ}$ Thomson side scattering at the wavelength of the interaction beam (Fig. 5). We observed a long on-axis line with two bright regions corresponding, probably, to two consecutive foci of the laser beam inside the plasma channel. When the laser power exceeds $2 \mathrm{TW}$ (that was about $4.6 P_{c}$

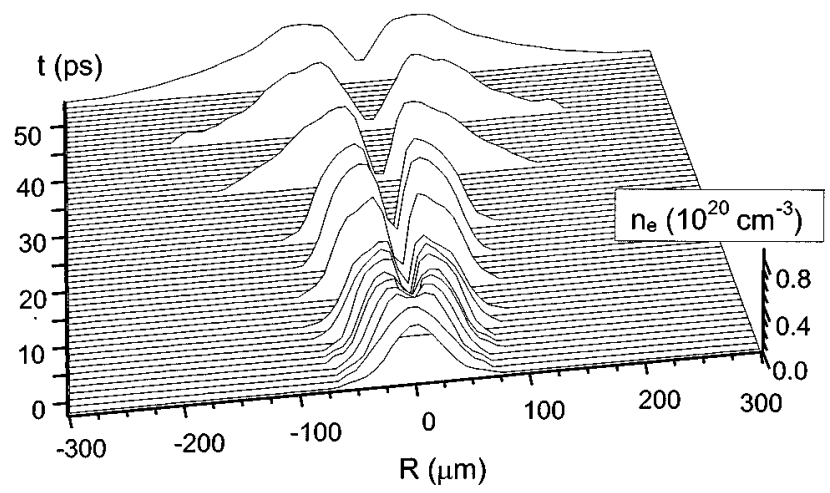

FIG. 8. Evolution of the radial distribution of electron density at the cross section $z=100 \mu \mathrm{m}$ inferred from the set of interferograms shown in Fig. 5. 


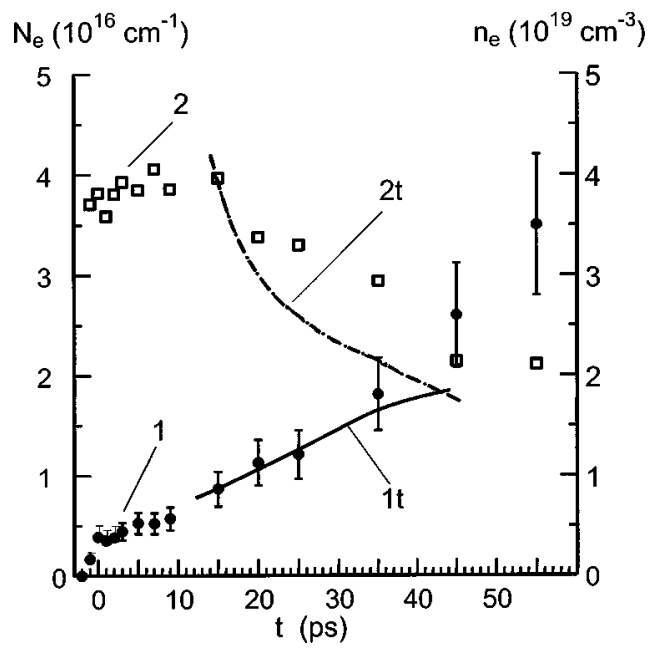

FIG. 9. Evolution of the linear electron density 1 (dots) and the mean electron density 2 (open squares) at the cross section $z$ $=100 \mu \mathrm{m}$. The corresponding theoretical curves $1 t$ and $2 t$ have been obtained from the model described in Sec. IV.

for relativistic self-focusing for our experimental conditions) the channel length extends to $1 \mathrm{~mm}$. We also observed that the scattered light intensity was decreased in the direction of laser beam propagation.

The radial expansion velocities in the cross section $z$ $=100 \mu \mathrm{m}$ for different interference phase shifts are shown in Fig. 6 . The velocity of the plasma radial expansion is found to be high: $4.1 \times 10^{8} \mathrm{~cm} / \mathrm{s}$ for the phase shift of the 0.2 line, and $3.5 \times 10^{8} \mathrm{~cm} / \mathrm{s}$ for the phase shift of the 0.5 line. The two-dimensional reconstruction of the electron density profile was performed using the Abel inversion procedure, with an assumption of the axial symmetry of a plasma, and attributing all changes of the refraction index $\mu$ to free electrons: $\mu=\sqrt{1-n_{e} / n_{c p}}$, where $n_{e}$ is the local electron density and

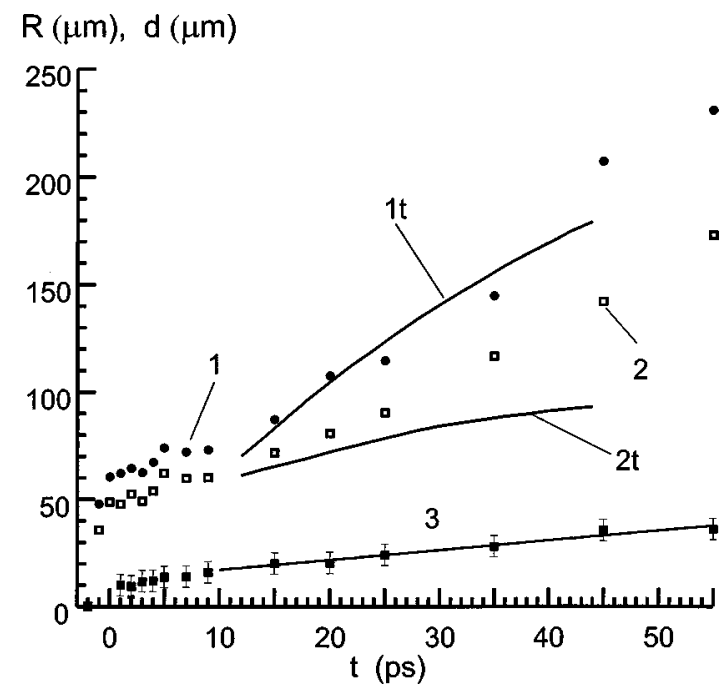

FIG. 10. Evolution of the plasma radius for the cross section $z$ $=100 \mu \mathrm{m}$. Experimental points 1 (dots) and 2 (open squares) show the positions of electron densities $5 \times 10^{18}$ and $1.5 \times 10^{19} \mathrm{~cm}^{-3}$, respectively. The points 3 (solid squares) show the evolution of the plasma channel diameter. The theoretical curves $1 t$ and $2 t$ corresponding to the experimental results 1 and 2 have been obtained from the model described in Sec. IV.

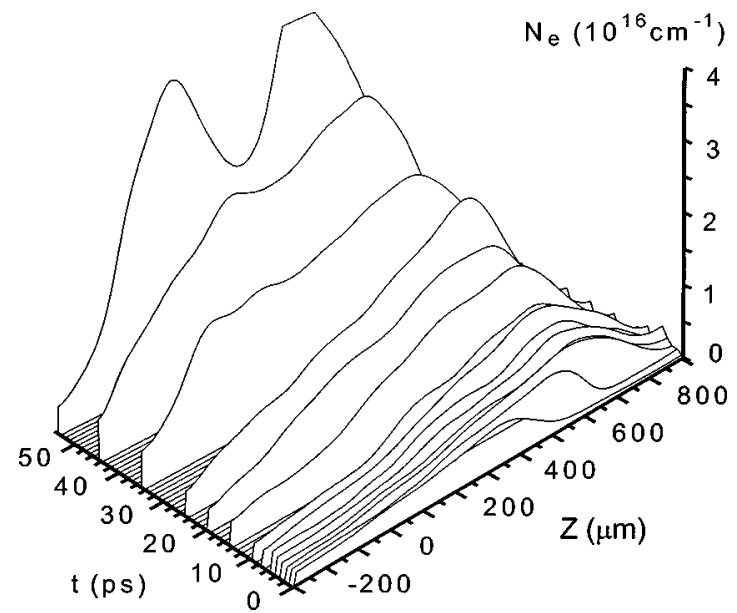

FIG. 11. The axial distribution of linear electron density for different moments of time inferred from the set of interferograms shown in Fig. 5.

$n_{c p}=\pi m_{e} c^{2} / e^{2} \lambda^{2}$ is the critical density for the probe laser wavelength. The inferred electron density profile for time moment $t=35 \mathrm{ps}$ is presented in Fig. 7. The maximum electron density is $\sim 7.6 \times 10^{19} \mathrm{~cm}^{-3}$ at a radius of $\sim 20 \mu \mathrm{m}$ and the estimated depth of the plasma channel is up to $80-90 \%$. The radius of the plasma channel is less than $10 \mu \mathrm{m}$, which is close to the resolution limit of our diagnostics. The accuracy of measurement of the channel depth is limited by the Abel inversion procedure.

Now we discuss the temporal behavior of the plasma channel. Although only one image per laser shot has been taken, the laser parameters and gas jet conditions were highly reproducible, and the time delay between the interaction and probe pulses was controlled with an accuracy better than $0.1 \mathrm{ps}$. The reproducibility of results has been verified by comparing the images taken from different shots for a same delay time. Because of these circumstances we consider pictures taken at different shots with different time delays as a representative history of plasma evolution in a single shot. The dynamics of electron density profile at $z$ $=100 \mu \mathrm{m}$ is shown in Fig. 8. The energy fluctuation between different shots was within $8 \%$ around the average energy of $1.7 \mathrm{~J}(4.3 \mathrm{TW})$. The electron density gradient at channel walls reaches the value of $5 \times 10^{22} \mathrm{~cm}^{-4}$ at a time of $7 \mathrm{ps}$, and remains practically the same up to $55 \mathrm{ps}$. Since the characteristic electron density was about $5 \times 10^{19} \mathrm{~cm}^{-3}$, this density gradient corresponds to the radial scale length of about $10 \mu \mathrm{m}$.

The evolution of linear electron density [the number of electrons per unit length is $\left.N_{e}=2 \pi \int r d r n_{e}(r)\right]$ at $z$ $=100 \mu \mathrm{m}$, and the evolution of mean electron density in the same cross section, are presented in Fig. 9. After the initial phase of fast gas ionization by the laser (from -2 to $0 \mathrm{ps}$ ), the number of electrons remains practically constant (from 0 to $9 \mathrm{ps}$ ), then the gas ionization starts again. At the same time the average electron density begins to decrease. There was no laser radiation at that time; therefore, this secondary ionization can be produced only by the laser-accelerated plasma.

The above described features of the plasma production are in agreement with the temporal behavior of the plasma radius 
and channel diameter, which are shown in Fig. 10. The plasma radius remains approximately constant from 0 to 10 $\mathrm{ps}$, then the plasma begins to expand radially. If we define the plasma edge as a region where the electron density is equal to $5 \times 10^{18} \mathrm{~cm}^{-3}(13 \%$ single ionization of He gas with $n_{\mathrm{He}}=4 \times 10^{19} \mathrm{~cm}^{-3}$ ), then the radial expansion velocity, $V_{0.5}$ is equal to $\sim 3.8 \times 10^{8} \mathrm{~cm} / \mathrm{s}$. Plasma regions with a higher degree of ionization, $1.5 \times 10^{19} \mathrm{~cm}^{-3}$ (40\% single ionization), expand with a lower velocity, $V_{1.5} \sim 2.5 \times 10^{8} \mathrm{~cm} / \mathrm{s}$. The channel diameter increases in time much more slowly than the plasma radius. The typical value of radial velocity of channel expansion is $\sim 4 \times 10^{7} \mathrm{~cm} / \mathrm{s}$.

A two-dimensional distribution of linear electron density versus an axial position at different moments of time is presented in Fig. 11. This distribution shows the same characteristic plasma behavior. Up to a time of $10 \mathrm{ps}$ the axial profile of linear density corresponds to the shape of the laser ionization cone in the gas. After 10 ps one can see a fast increase of linear density due to the ambient gas ionization. The fastest rate of plasma production occurs at two cross sections $z=100$ and $600 \mu \mathrm{m}$, where $d N_{e} / d t \approx 4 \times 10^{14}$ $\mathrm{cm}^{-1} \mathrm{ps}^{-1}$. The minimum of the plasma production rate is at the cross section $z=350 \mu \mathrm{m}$. Below, we present a theoretical model of the ambient gas ionization, and employ it to retrieve the parameters of accelerated ions and a laser channel.

\section{PHYSICAL MODEL}

\section{A. Qualitative considerations}

Although the observations presented in Sec. II B definitely indicate the formation of a long narrow channel on the laser axis, one has to explain the origin of the secondary gas ionization $10 \mathrm{ps}$ after the laser pulse, and relate the observed features of the electron density distribution to the characteristics of the laser pulse. Hence we begin with an identification of the origin of the secondary gas ionization. It has been observed that two distinct features occur in the experimental data: a 10-ps delay of ionization, and an approximately constant high velocity propagation of the ionization front through the ambient gas. Diffusive processes like electron thermal transport and radiative transport cannot explain these features of gas ionization. Indeed, for both these processes, the temperature equation predicts the formation of a steep heat front propagating into a cold gas medium with a velocity that decreases with time as $t^{-\alpha}$ where $\alpha=\frac{7}{9}$ for the classical Spitzer-Härm electron heat conductivity and $\alpha=\frac{6}{7}$ for the radiative transport, respectively [30]. These analytic time scalings are in apparent contradiction with the observations.

The nonlocal electron heat transport [31] might be responsible for the fast ionization wave propagation, if we assume that the electron temperature is above few $\mathrm{keV}$ and, hence, that the electron mean free path is about $100 \mu \mathrm{m}$ or more. However, such electrons have a very low ionization cross section, below $10^{-17} \mathrm{~cm}^{2}$ [32], which is not enough to explain the observed high ionization rate. Also, the experiment by Krushelnik et al. [9] suggested that the actual electron temperature in the laser channel is less than $1 \mathrm{keV}$.

The ionization can also not be explained by a shock wave, because of the large propagation velocity, which is orders of magnitude larger than the acoustic velocity in a helium gas. This implies a strong gas compression behind the shock, which is not supported by shadow and interferometric images shown above (cf. Fig. 4).

Therefore, we conclude that only free streaming particles could explain the observed features of the ionization front. At this moment gas ionization by an electron stream should be excluded. This is because electrons with velocities $\sim 4$ $\times 10^{8} \mathrm{~cm} / \mathrm{s}$ (an energy of about $45 \mathrm{eV}$ ) have very short mean free paths in helium at normal pressure, about $1 \mu \mathrm{m}$, while observations show the ionization front propagates more than hundred microns in the gas. Hence we identify the ambient gas ionization with the propagation of fast helium ions. These ions have been expelled from the laser-produced plasma channel and have an energy of more than $300 \mathrm{keV}$. The mean free path of such ions in a normal pressure gas is few millimeters, and they penetrate through a gas as projectiles, experiencing only small angle elastic scattering and losing their energy to the neutral atoms via excitation and ionization. Certainly these ions cannot propagate alone; they carry an appropriate number of free electrons with them in order to compensate for the space charge. However, these escorting electrons have a minor contribution to the gas ionization and excitation. The velocity of such fast ions is comparable to the velocities of bounded electrons in the helium atom, and therefore ions efficiently ionize the ambient gas. Their ionization cross section is about $3 \times 10^{-16} \mathrm{~cm}^{2}$ [33], and, therefore, each ion produces approximately one electron-ion pair on a $1-\mu \mathrm{m}$ path in the helium gas with a density of $\sim 4 \times 10^{19} \mathrm{~cm}^{-3}$. Such fast ions also have a large excitation cross-section, about $2 \times 10^{-16} \mathrm{~cm}^{2}$ [34] for the transition $1 s \rightarrow 2 p$. They are much more efficient in helium gas ionization than electrons, which have a maximum ionization cross section about $3 \times 10^{-17} \mathrm{~cm}^{2}$ [32].

It is evident that the ionization front propagates with a velocity comparable to, but less than, the velocity of the fastest ions. Also, due to the radial divergence of fast ion flow, their ionization efficiency decreases at larger radii; therefore, the areas with higher electron density expand with lower velocity decreasing with time. The hypothesis of gas ionization by fast ions also offers a natural explanation for the 10-ps ionization delay: it is the time required for the fast ions to penetrate through a plasma, with a radius of about 40 $\mu \mathrm{m}$. The electron density does not change during that time period, since there are no neutral atoms in this area. Such a plasma volume can clearly be seen in Fig. 4. It has been produced by the low intensity spatial wings of the laser light because of a relatively low threshold for helium multiphoton ionization, which is about $10^{15} \mathrm{~W} / \mathrm{cm}^{2}$ for the primary ionization and $\sim 10^{16} \mathrm{~W} / \mathrm{cm}^{2}$ for the secondary ionization $[15,27,28]$.

It is important to note that fast ion propagation through the ambient gas and the gas excitation and ionization cannot be explained in terms of the conventional ion hydrodynamics [19] for two reasons: first, the accelerated ions are almost collisionless and do not interact elastically with the ambient gas; second, the ions have an energy spectrum, which is defined by the acceleration mechanism, and, therefore, one has a multigroup ion flow that requires a kinetic treatment. One of the important consequences of the kinetic nature of the ion expansion is that the diameter of the evacuated plasma channel is defined approximately by the size of laser beam and does not increase with time. While in the hydrodynamic de- 
scription the diameter of the plasma channel proceeds to increase above the laser beam diameter [19], since the accelerated ions are supposed to exchange their momentum with ambient ions (or atoms) and they move together (as a snow plow) as long as the total initial ion energy will be converted into a work against the pressure force of ambient gas. Curve 3 in Fig. 8 demonstrates that indeed the radius of plasma channel does not change in time significantly, which supports the kinetic model.

\section{B. Coulomb explosion}

Now we turn our attention to the mechanism of the laserplasma coupling and ion acceleration. A qualitative picture of ion acceleration by the laser beam ponderomotive force was discussed in Ref. [35], and called the "Coulomb explosion,' since ions are accelerated by the electrostatic field of charge separation produced in a plasma when the electrons are expelled from the laser beam channel due to the radial component of the ponderomotive force. However, one requires a quantitative treatment for the experiment interpretation.

We consider a laser pulse interaction with an underdense plasma for the conditions where the laser frequency $\omega_{0}$ is much larger than the electron plasma frequency $\omega_{p}$ and the laser pulse duration $\tau$ is larger than the electron response time $1 / \omega_{p}$. Also, the spatial plasma scale and laser intensity scale are assumed to be larger than the laser wavelength $\lambda_{0}$. Then one can average the electron response over the laser period and consider electrons as a fluid which is influenced by the laser ponderomotive force

$$
\mathbf{F}_{p}=-m_{e} c^{2} \nabla \sqrt{1+a^{2} / 2}
$$

where $a=0.85 \times 10^{-9} \lambda_{0}[\mu \mathrm{m}] \sqrt{I\left[\mathrm{~W} / \mathrm{cm}^{2}\right]}$ is the normalized slowly varying vector potential of the laser field, and $I$ is the laser pulse intensity.

As mentioned above, the gas was preionized by the laser prepulse approximately 2 ps before the main pulse arrival, and the laser pulse rise time is much larger than that of the electron plasma period (which is few fs for our experimental parameters). Therefore, electrons respond adiabatically, and the ponderomotive force (1) is balanced by an electric field of charge separation $e \mathbf{E}=\mathbf{F}_{p}$. This electric field is applied to ions, and accelerates them during the time of the laser pulse passage. Ions continue to move after the passing of the laser pulse due to their own inertia. To support plasma quasineutrality, the electrons are forced to accompany the ions. Thus, for a short laser pulse the plasma cavity formation occurs in the ion evacuation time, which might be longer than the pulse duration.

If the pulse length $c \tau$ is larger than the pulse radius $r_{0}$, the radial component of the ponderomotive force dominates, and ions are accelerated radially. According to Eq. (1), the ion energy depends dramatically on the laser beam radius. Hence knowing the final energy of accelerated ions and the laser pulse duration and energy, one can deduce the radius of the laser beam and its on-axis intensity.

The process of self-focusing and channeling of a short relativistic laser pulse has been studied in detail elsewhere $[4,11,13,15]$. In our conditions the laser power exceeds the critical power for the relativistic self-focusing more than ten times. The theory and numerical simulations for similar conditions suggest that a substantial part of the laser power might be trapped in a narrow channel near the laser axis, and the channel length could be many times the Rayleigh length. We concentrate here on the processes that occur in a plasma after the laser channel has been formed. These are the ion acceleration, plasma channel formation, and subsequent ion interaction with the ambient gas. Doing so, we leave aside the process of laser beam self-focusing, and assume right away that a certain part of the laser pulse energy is already channeled. Then we approximate part of the pulse trapped in the channel with Gaussian functions in time and radius:

$$
I(r, t)=\frac{E_{0}}{\pi^{3 / 2} r_{0}^{2} \tau} \exp \left(-\frac{r^{2}}{r_{0}^{2}}-\frac{t^{2}}{\tau^{2}}\right),
$$

where $E_{0}$ is the trapped laser energy, $I_{0}=E_{0} / \pi^{3 / 2} r_{0}^{2} \tau$ is the on-axis laser intensity, and $r_{0}$ is the radius of the laser channel. Keeping in mind possible variations in the channel radius and losses of the channeled energy, we can consider $E_{0}$ and $r_{0}$ as functions of $z$-the coordinate along the channel axis.

\section{Collisionless ion kinetics}

To formulate quantitatively the physical model of plasma channel formation, we supplement the quasistationary description of the relativistic electrons by the kinetic description of the cold collisionless nonrelativistic ions with the mass $m_{i}$ and charge $Z e$. Assuming that ions move only in a radial direction, the kinetic equation for the ion distribution function $f_{i}$ reads:

$$
\frac{\partial f_{i}}{\partial t}+v_{i} \frac{\partial f_{i}}{\partial r}+\frac{Z}{m_{i}} F_{p} \frac{\partial f_{i}}{\partial v_{i}}=0 .
$$

The last term in the left hand side accounts for the ion acceleration due to the electric field produced by the ponderomotive force (1).

Equation (3) has been solved numerically by the particlein-cell method [36], and we present the results below. The characteristic energy of the accelerated ion, $\boldsymbol{\epsilon}_{i}=m_{i} v_{i}^{2} / 2$, can be estimated for the case of a short laser pulse when the pulse length $\tau$ is shorter than the ion response time $r_{0} / v_{i}$. The radial ion acceleration, according to Eq. (1), is $\left(Z m_{e} / m_{i}\right) c^{2} \nabla_{r} \sqrt{1+a^{2} / 2}$, so the radial velocity of the ions after the laser pulse ends reads

$$
v_{i}=-\frac{Z m_{e}}{m_{i}} c^{2} \frac{\partial}{\partial r} \int d t \sqrt{1+a^{2} / 2}
$$

This formula predicts the ion energy spectrum $0<\epsilon_{i} \leqslant \epsilon_{\max }$ with the energy cutoff

$$
\epsilon_{\max } \sim \frac{Z^{2} m_{e}^{2} c^{4}}{16 m_{i} r_{0}^{2}} \frac{a_{0}^{4} \tau^{2}}{1+a_{0}^{2} / 2} \approx 3 \frac{Z^{2}}{A} \frac{\tau^{2}}{r_{0}^{2}} \frac{a_{0}^{4}}{1+a_{0}^{2} / 2} \mathrm{MeV},
$$

where $a_{0} \approx 3.6 \sqrt{E_{0} / \tau} \lambda_{0} / r_{0}$ is the maximum vector potential of the interaction laser pulse, and $A$ is the ion mass number. Here $E_{0}$ is measured in $\mathrm{J}, \tau$ in ps, $\lambda_{0}$ and $r_{0}$ in $\mu \mathrm{m}$. According to this formula, in the relativistic limit, $a_{0} \gtrsim 1$, the ion 
energy is approximately proportional to the pulse energy and pulse length, and inversely proportional to the fourth power of the laser beam radius. This high power radius dependence can be used to resolve an inverse problem: to estimate the laser channel radius knowing the ion energy.

The total energy of accelerated ions per unit length of the laser channel can be estimated as follows:

$$
\frac{d \mathcal{E}}{d z}=\int 2 \pi r d r n_{i} \frac{m_{i} v_{i}^{2}}{2} \approx 0.08 r_{0}^{2} n_{a} \epsilon_{\max } \mathrm{J} / \mathrm{cm}
$$

where the ion density $n_{i}$ (that is effectively the atomic density of gas $n_{a}$ ) is in units $10^{19} \mathrm{~cm}^{-3}, \epsilon_{\max }$ is in $\mathrm{MeV}$, and $r_{0}$ is in $\mu \mathrm{m}$. For a relativistically strong laser pulse the energy deposited in the accelerated ions could be an important channel of the laser energy losses. Although the absorption length $L_{a}=E_{0} /(d \mathcal{E} / d z)$, calculated from Eq. (6), is much larger than the Rayleigh length, the laser pulse energy losses for the ion acceleration might be more significant than the inverse Bremsstrahlung absorption, and could be comparable to the losses due to the stimulated Raman scattering. In the relativistic limit, $a_{0} \gtrsim 1$, according to Eqs. (5) and (6), the laser pulse energy losses due to the ion acceleration scale linearly with the laser pulse energy and pulse length, and are inversely proportional to the square of the beam radius.

\section{Refraction of a probe laser beam in a plasma}

The refraction coefficient for the electromagnetic wave in a fully ionized underdense plasma is determined by free electrons. Thus a simple expression $\mu=\sqrt{1-n_{e} / n_{c p}} \approx 1$ $-n_{e} / 2 n_{c p}$ for a plasma refraction index for a probe laser light can be used to infer the electron density from interferometric measurements. However, this is not a case for a partly ionized gas, where the excited atoms and incompletely stripped ions might also contribute to a plasma refractivity. (The contribution of completely stripped ions can be neglected due to the high ion-to-electron mass ratio.) The contribution of an excited atom or ion, in a given $i$ th excitation state, to the plasma refraction index depends on the wavelength $\lambda_{j i}$ of allowed electron transitions $i \rightarrow j$ and on the corresponding oscillator strength $f_{j i}$ :

$$
\mu(\lambda)=1-\frac{e^{2} \lambda^{2}}{2 \pi m_{e} c^{2}}\left(n_{e}+\sum_{i, j} \frac{f_{j i} n_{\mathrm{ai}}}{1-\lambda^{2} / \lambda_{j i}^{2}}\right),
$$

where $\lambda$ is the probe wavelength, and $n_{\mathrm{ai}}$ is the excited atomion density in the $i$ th state.

Free electrons always produce a negative contribution to the refractive index. The contribution of bounded electrons [the second term in parentheses in Eq. (7)] could have a different sign which depends on the relation between the transition wavelength and the probe laser light wavelength. The plasma refraction index dramatically increases if the probe wavelength is close to the transition wavelength. This case of the resonance refraction is frequently used for diagnostics of excited atoms and ions. In our conditions the probe wavelength is nonresonant, and the contribution of excited atoms and free electrons to the refraction index could be comparable. It follows from Eq. (7) that the Abel inversion procedure allows one to infer the cumulative contribution the free electrons and the bounded electrons of excited atoms,

$$
n_{e f f}=n_{e}+\sum_{i, j} \frac{f_{j i} n_{a i}}{1-\lambda^{2} / \lambda_{j i}^{2}},
$$

but not the density of free electrons alone. Such an effective electron density can be significantly different from $n_{e}$ and in some cases could even be negative.

\section{E. Excitation and ionization of ambient gas}

The energy spectrum of accelerated ions obtained from the solution to Eq. (3]) has been used in calculations of the secondary electron population and the excited He atoms. We neglect the momentum exchange in $\mathrm{He}^{2+}-\mathrm{He}$ collisions, since the energy loss per each electron-ion pair production is on the order of the ionization potential, which is much less than the ion energy. Hence the equations for the concentration of secondary free electrons, $n_{e}$, and excited $\mathrm{He}$ atoms, $n_{*}$, read

$$
\frac{\partial n_{\alpha}}{\partial t}=n_{\mathrm{He}} \int_{0}^{v_{\max }} d v_{i} \sigma_{\alpha}\left(v_{i}\right) v_{i} f_{i}\left(v_{i}\right),
$$

where $\alpha=e, *$ for free electrons and excited atoms, respectively, $n_{\mathrm{He}}$ is the density of neutral helium atoms, and $\sigma_{\alpha}$ is the cross section of the ionization (excitation) of neutral $\mathrm{He}$ atoms by the $\mathrm{He}^{2+}$ projectiles and electrons accompanying them. These cross sections have been taken from Refs. [3234], and interpolated by analytic functions.

The ionization cross section of $\mathrm{He}$ by $\mathrm{He}^{2+}$ projectiles is taken from Refs. [33], and approximated as follows:

$$
\sigma_{e}(v)=71.4 \frac{\ln \left(2.72+0.07 v^{2}\right)}{v^{2}+625 / v^{2.8}} \exp \left(-\frac{0.5}{v}\right) \text {, }
$$

where the ion velocity $v$ is in $10^{8}-\mathrm{cm} / \mathrm{s}$ units, and $\sigma_{e}$ is in $10^{-16}-\mathrm{cm}^{2}$ units. The maximum of this cross section is 3.1 $\times 10^{-16} \mathrm{~cm}^{2}$ for ions with a velocity $4.5 \times 10^{8} \mathrm{~cm} / \mathrm{s}$. Among all possible excitations for He atoms, we choose to consider only one transition, $1 s \rightarrow 2 p$ (from the ground state $1{ }^{1} S$ to the state $2{ }^{1} P$ ), which has a much larger cross section than any other transition. However, we increase the corresponding cross section by $20 \%$ to take into account, in a rough way, all other transitions. The excitation cross section for the transition $1 s \rightarrow 2 p$ has the following analytical approximation for $\mathrm{He}$, suggested in Ref. [34]:

$$
\sigma_{*}(v)=91 \frac{\ln \left(2.72+0.037 v^{2}\right)}{v^{2}+1135 / v^{2.3}} \exp \left(-\frac{0.67}{v}\right) .
$$

This achieves a maximum of $2.1 \times 10^{-16} \mathrm{~cm}^{2}$ at an ion velocity of $6 \times 10^{8} \mathrm{~cm} / \mathrm{s}$. Assuming that the number and energy of $\mathrm{He}^{2+}$ projectiles do not change with time, we neglect the process of the charge exchange in $\mathrm{He}^{2+}-\mathrm{He}$ collisions which effectively reduces the number of fast $\mathrm{He}^{2+}$ ions and consequently reduces the secondary ionization. Although the charge exchange dominates the ionization and excitation cross sections at low ion velocities, $v_{i} \leqslant 1 \times 10^{8} \mathrm{~cm} / \mathrm{s}$, its 
contribution is several times less for the velocities above 3 $\times 10^{8} \mathrm{~cm} / \mathrm{s}$ and its incorporation would significantly complicate our model.

The electrons are assumed to have a Maxwellian distribution, and have been included in Eq. (9) for free-electron production in terms of a constant ionization rate $\left\langle\sigma_{e}^{(e)} v_{e}\right\rangle \approx 2$ $\times 10^{-8} \mathrm{~cm}^{3} / \mathrm{s}$ [32] which almost does not depend on the electron temperature, if it is in the range from $100 \mathrm{eV}$ to several $\mathrm{keV}$. This ionization rate has been multiplied by a factor of 2 since each ion is escorted by two electrons. We suspended the electron ionization contribution for small ion velocities, $v_{i} \lesssim 1 \times 10^{8} \mathrm{~cm} / \mathrm{s}$, since the charge exchange is supposed to decrease the number of such ions, and electrons cannot propagate far enough without ions. Our analysis shows that electron contribution to the free electron production could be as much as $30 \%$. The electron contribution to the excitation of ambient gas has been neglected.

For the $\mathrm{He}$ atom in the state $2{ }^{1} P$ there are four most important transitions which we took into account while calculating its response to the probe laser field. Those are [37] the transition to the ground state $1{ }^{1} S\left(\lambda_{1}=0.5843 \mu \mathrm{m}, f_{1}\right.$ $=0.276)$, the transition to the state $2{ }^{1} S\left(\lambda_{2}=20.58 \mu \mathrm{m}\right.$, $\left.f_{2}=0.376\right)$, the transition to the state $3{ }^{1} D\left(\lambda_{3}=6.678 \mu \mathrm{m}\right.$, $\left.f_{3}=0.711\right)$, and the transition to the state $4{ }^{1} \mathrm{D}\left(\lambda_{4}\right.$ $\left.=4.92 \mu \mathrm{m}, f_{4}=0.121\right)$. Combining all these transitions for a probe wavelength $\lambda=1.053 \mu \mathrm{m}$, one finds from Eq. (7) that excited atoms make a contribution similar to that of free electrons with the coefficient 1.1. Therefore, the effective electron density that has been measured in the experiment can be written as follows: $n_{e}^{\text {eff }}=n_{e}+1.1 n_{*}$. Keeping in mind that the ionization and excitation cross-sections are comparable in magnitude, we conclude that excited He atoms contribute as much as $50 \%$ to the observed secondary "ionization."

\section{MODELING OF THE EXPERIMENT}

Equations (3) and (9) have been solved simultaneously with the input parameters taken from the experiment: gas density, $n_{\mathrm{He}}=(3-5) \times 10^{19} \mathrm{~cm}^{-3}$, pulse length $\tau=0.24 \mathrm{ps}$ (which corresponds to 400-fs FWHM), and pulse energy $E_{0}=1-1.5 \mathrm{~J}$ (we assume that approximately $60-80 \%$ of the incident energy has been trapped in the laser channel). The radius of the plasma channel is $r_{0}=2.5-3 \mu \mathrm{m}$, and the radius of the preionized region is $r_{p l}=35-45 \mu \mathrm{m}$. Two parameters have been varied in order to fit the experimental data: the laser channel radius $r_{0}$ and the initial density of the helium gas, $n_{\mathrm{He}}$. The first of them controls the maximum energy of the accelerated ions, while the second one controls the production rate of secondary electrons. The radius of the preionized region has been adjusted in each run after $n_{\mathrm{He}}$ has been chosen in order to fit the measured total number of electrons just after the end of the main laser pulse, $N_{e} \approx 0.4$ $\times 10^{16} \mathrm{~cm}^{-1}$, at time $t=1-3 \mathrm{ps}$.

The dynamics of plasma channel formation is demonstrated in Fig. 12, where the radial ion density distribution is shown as a function of time. A sharp narrow outer peak in the density distribution indicates the radial position of fastest ions. There is also a density peak behind the front at the edge of the channel which is the contribution of slower ions.

The first goal of simulations was to fit the observed rate of secondary plasma production, $d N_{e} / d t$, and the velocity of

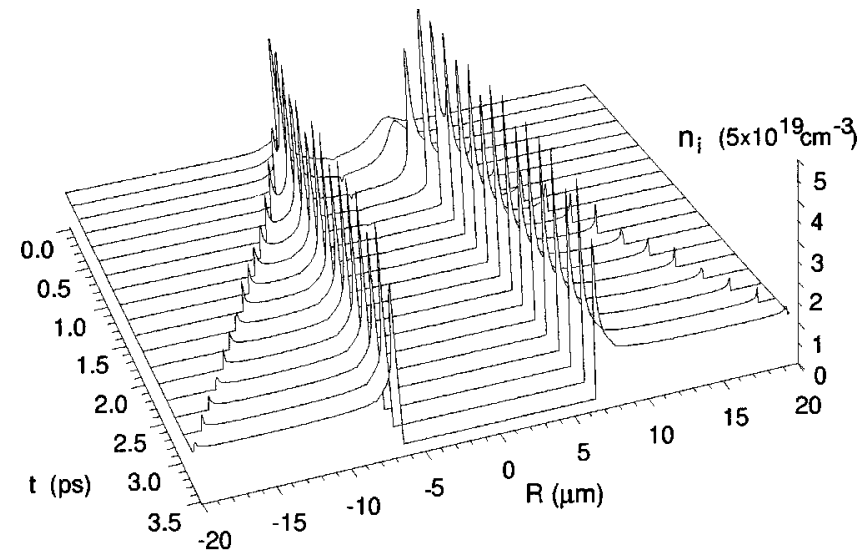

FIG. 12. Modeling of the evolution of ion density in the cross section $z=100 \mu \mathrm{m}$ for the experimental conditions: laser pulse energy inside the channel $1.36 \mathrm{~J}$, pulse duration $400 \mathrm{fs}$, laser channel radius $2.6 \mu \mathrm{m}$, and ambient $\mathrm{He}$ gas density $5 \times 10^{19} \mathrm{~cm}^{-3}$.

plasma expansion with a given electron density for the cross section $z=100 \mu \mathrm{m}$. The latter, according to the explanations of Sec. III D, is actually the effective electron density, $n_{\text {eff }}$ $=n_{e}+1.1 n_{*}$. It was found from the simulations that the plasma production rate and the plasma expansion velocity are most sensitive to the background gas density. Both of them increase dramatically as $n_{\mathrm{He}}$ increases. There is also a dependence on $r_{0}$, but it is not so pronounced because the decrease in laser channel radius has a two sided effect: it increases the ion velocity, on the one hand, and correspondingly increases the ionization-excitation cross section; however, on the other hand, it decreases the total number of accelerated ions and, therefore, decreases the plasma production rate.

It turned out to be impossible to achieve the observed parameters with the gas density of $3.5 \times 10^{19} \mathrm{~cm}^{-3}$ : the plasma production rate and the expansion velocities $V_{0.5}$ and $V_{1.5}$ (the velocities of plasma regions with densities of 0.5 $\times 10^{19} \mathrm{~cm}^{-3}$ and $1.5 \times 10^{19} \mathrm{~cm}^{-3}$, correspondingly) were several times below the observed values $\left(d N_{e} / d t \approx 1.5\right.$ $\times 10^{14} \mathrm{~cm}^{-1} \mathrm{ps}^{-1}, V_{0.5} \approx 1.5 \mu \mathrm{m} / \mathrm{ps}$, and $V_{1.5} \approx 0.4 \mu \mathrm{m} / \mathrm{ps}$ for $E_{0}=1.36 \mathrm{~J}$ and $\left.r_{0}=2.6 \mu \mathrm{m}\right)$. A reasonable agreement with the experimental data can be achieved in the model with a gas density of $5 \times 10^{19} \mathrm{~cm}^{-3}$. Correspondingly, the following set of input parameters has been chosen: $n_{\mathrm{He}}=5 \times 10^{19}$ $\mathrm{cm}^{-3}, r_{p l}=35 \mu \mathrm{m}, E_{0}=1.36 \mathrm{~J}$, and $r_{0}=2.6 \mu \mathrm{m}$. With these parameters the plasma production rate is $d N_{e} / d t \approx 3.6$ $\times 10^{14} \mathrm{~cm}^{-1} \mathrm{ps}^{-1}$, the expansion velocity is $V_{0.5} \approx 3.8 \mu \mathrm{m} / \mathrm{ps}$, and the fastest ions propagate with the velocity $V_{\max }=5.2 \mu \mathrm{m} / \mathrm{ps}$ (energy $550 \mathrm{keV}$ ). The assumed radius of the laser channel also agrees with the radii of beams obtained in modeling of the relativistic laser beam selffocusing $[4,11,13,15]$. The dependencies of the gas ionization rate, $d N_{e} / d t$, and the velocities, $V_{0.5}$ and $V_{\max }$, on the channel radius $r_{0}$ are shown in Fig. 13.

According to Fig. 12, the formation of plasma channel begins during the propagation of the interaction pulse: there is a depression at the beam axis and a compression outward at a time of the laser pulse maximum $t=0$. The time of channel formation is about $2 \mathrm{ps}$. After that time the channel is completely formed and the expelled ions empty the central region $r<5 \mu \mathrm{m}$. The fastest ions are propagated away from 


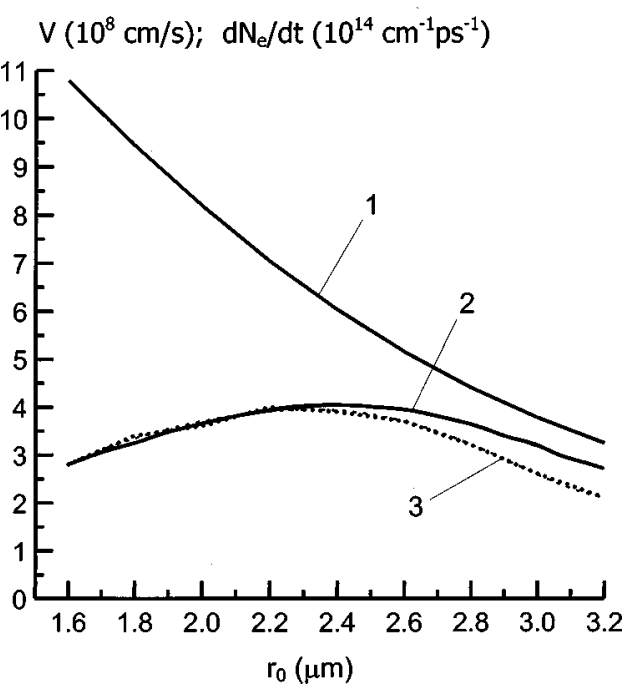

FIG. 13. The dependence of the maximum ion velocity $V_{\max }$ (curve 1), the velocity $V_{0.5}$ (curve 2 ), and the gas ionization rate, $d N_{e} / d t$ (curve 3 , dashed line) on the channel radius for parameters of the experiment.

the channel, while the slow ions form a compression ring at the border of the plasma channel. The maximum compression is 6-7 times that of the initial density at time about 10 ps and then it gradually decreases. The thickness of the compression region is less than $1 \mu \mathrm{m}$, which is below the experimental resolution. This explains a low experimentally measured density compression (less than two times). The radius of the plasma channel is $7 \mu \mathrm{m}$ at $4 \mathrm{ps}$. That is three times larger than the minimal laser focal spot radius at half the maximum intensity. At later times the plasma channel radius increases very slowly; it also increases with the laser beam energy.

Such dynamics of plasma cavitation is in dramatic contrast with the hydrodynamic calculations reported in Ref. [19]. We see that the radius of the plasma channel is set up within a few ps, and does not change significantly later on. However, in hydro simulations the channel has a much larger radius and it expands for a much longer time. The hydrodynamic model also predicts a different behavior for the compression wave: the plasma compression is much larger in the hydro model, and it increases with time. The kinetic predictions of the channel dynamics agree qualitatively with the experiment. The channel diameter indeed sets up to 10-15 $\mu \mathrm{m}$ within first few picoseconds, and then demonstrates a much slower increase up to $30 \mu \mathrm{m}$. The latter feature is out of the scope of our model for two possible reasons: the laser beam probably has non-Gaussian wings, which push ions at larger radial distances, and slow ions with energies below 10 $\mathrm{keV}$ cannot be treated as collisionless particles. The mean free path of such ions with respect to ion-electron collisions is about $1 \mu \mathrm{m}$. They transfer their momentum to ambient atoms and provide an additional, hydrodynamiclike, source for the channel expansion.

The ion energy distribution $N_{i}\left(\epsilon_{i}\right)=2 \pi \int r d r f_{i}$ is shown in Fig. 14 for $t=0$ ps (the maximum of the laser pulse) and for $t=2 \mathrm{ps}$. For later time moments this distribution does not change because we neglect ion energy losses for gas ionization and excitation. Since the function $N_{i}$ represents the ion distribution function integrated over the radius, particles with

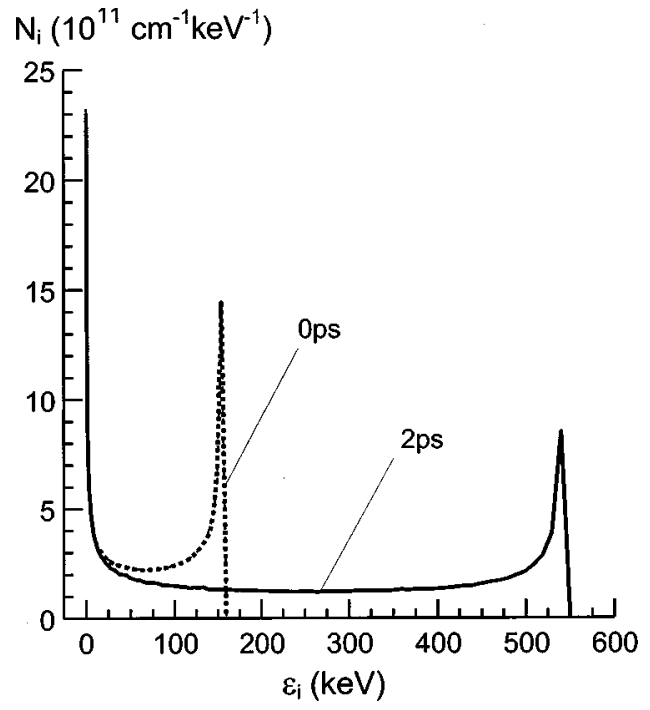

FIG. 14. Energy distribution of fast ions at two time moments $t=0$ and 2 ps. The parameters of modeling are presented in Fig. 12.

different energies are actually located at different distances from the axis. The ion distribution has a typical shape with two maxima at low and high energies with a distinct cutoff at the maximum energy $\epsilon_{\max }$.

Now we will discuss the dynamics of gas ionization and excitation. Theoretical curves for the time dependence of the linear electron density, $N_{e}(t)$, and the average effective electron density, $n_{e}(t)$, inside the radius $R_{0.5}$ are shown in Fig. 9. One can see a good qualitative agreement, although the model predicts a somewhat smaller plasma production rate, which does not increase at later times. This discrepancy could probably be attributed to slow collisional ions, which we do not account for in our simple kinetic model. The temporal evolution of the average electron density for $t>10 \mathrm{ps}$ is also in agreement with observations, although the model demonstrates an increase of the mean electron density during first the $10 \mathrm{ps}$, which is not seen in the experiment. This could be a consequence of our model's assumption of the distribution of an initial plasma density. Some amount of neutral He atoms have been left within the radius $40-50 \mu \mathrm{m}$ in the model; therefore, the secondary ionization takes place before the plasma radius starts to increase.

In Fig. 10 we show the time dependence of the radial positions of the regions with the given electron density: 1.5 $\times 10^{19}$ and $0.5 \times 10^{19} \mathrm{~cm}^{-3}$, for $R_{1.5}$ and $R_{0.5}$, correspondingly. The corresponding velocities $V_{j}=d R_{j} / d t$ change in time. They achieve their maxima during the initial time moments, and then gradually decrease. The temporal behavior and magnitudes of $R_{j}$ are similar to that in the experiment, although the experiment does not demonstrate a tendency of deceleration at later times. The average velocity of lowest density region at time $20-30 \mathrm{ps}, V_{0.5}=3.6 \times 10^{8}-\mathrm{cm} / \mathrm{s}$, is approximately 1.5 times less than the maximum ion velocity, while the higher density propagates much slower, $V_{1.5}=1.3$ $\times 10^{8} \mathrm{~cm} / \mathrm{s}$. We note that the model predicts smaller expansion velocity for plasma with $n_{\text {eff }}=1.5 \times 10^{19}-\mathrm{cm}^{-3}$ due to the reason we mentioned above (collisions of slow ions, which are not taken into account in our model).

We found that the model is very instructive in understanding the axial channel characteristics. The axial dependence 


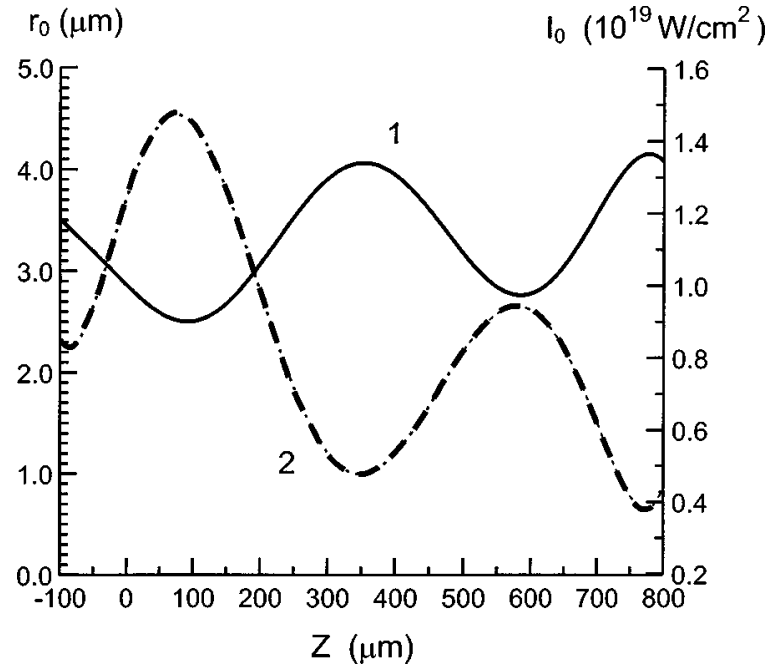

FIG. 15. Axial dependence of the laser channel radius (curve 1, solid line) and on-axis laser intensity (curve 2, dashed line) for parameters of the experiment.

can be introduced in our one-dimensional model in terms of parametric dependence of the laser pulse energy $E_{0}(z)$ and the laser channel radius $r_{0}(z)$ on the axial coordinate $z$. We assume an exponential decrease of $E_{0}$ with $z$ that corresponds to $60 \%$ laser energy loss on 1-mm length (which is the diameter of gas jet). The comparison of $V_{0.5}$ calculated from the model with the experimentally measured velocity of plasma expansion allows one to find an axial dependence of the laser channel radius, $r_{0}(z)$. Knowing the laser energy in a given cross section, one also finds the on-axis laser intensity $I_{0}(z)$. The results of this reconstruction of the laser channel characteristics in a plasma are shown in Fig. 15. One can see two regions of laser channel compression at $z=100$ and $600 \mu \mathrm{m}$, which are in agreement with the Thomson scattering data shown in Fig. 5. We infer that the maximum value of laser intensity in the plasma channel exceeds the vacuum intensity by almost three times.

Knowing the axial dependence of the laser energy, $E_{0}(z)$, and the laser channel radius $r_{0}(z)$, one can deduce the axial characteristics of fast ion generation. The fastest ions are generated from regions of tight focusing at $z=100$ and 600 $\mu \mathrm{m}$. Integrating the total energy of accelerated ions per unit length, $d \mathcal{E} / d z$, over the laser propagation length, we found

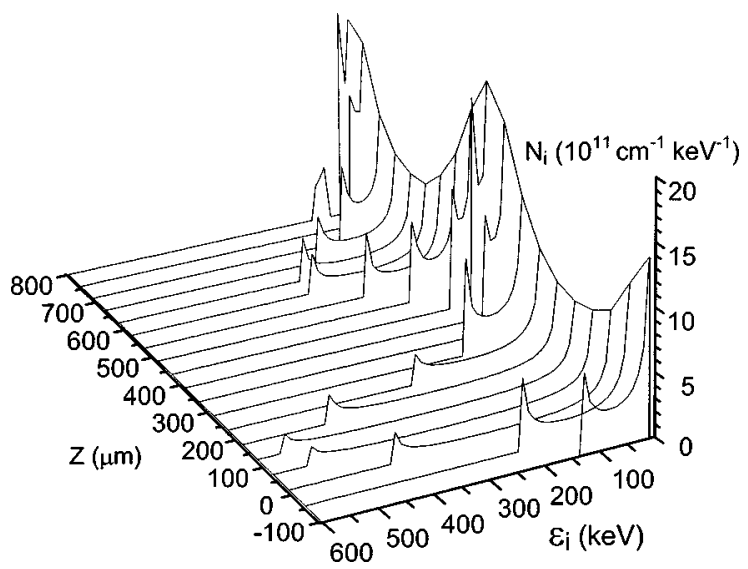

FIG. 16. Energy distribution of fast ions at different cross sections for parameters of the experiment.

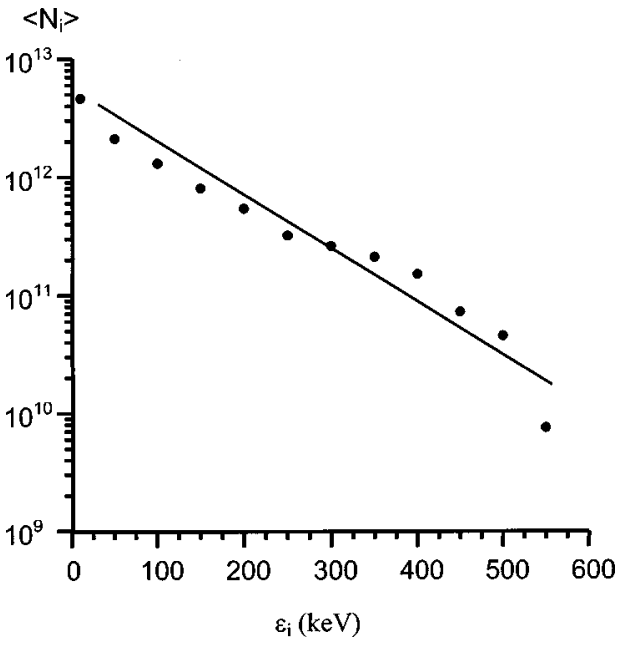

FIG. 17. Total number of fast ions with energies exceeding $\epsilon_{i}$ integrated over the laser channel length for parameters of the experiment. The solid line is the Maxwellian function with a temperature of $80 \mathrm{keV}$.

that about $65 \mathrm{~mJ}$ (or $\approx 5 \%$ of the laser energy trapped in the channel) is contained in the fast ions.

The ion energy distribution $N_{i}\left(\epsilon_{i}, z\right)$ is presented in Fig. 16. It is similar to the late time distribution shown in Fig. 14, but the cutoff of ion energy changes along the axis. Two maxima of the cutoff energy correspond to two consecutive foci of the laser beam. Although the ion energy distribution in each cross section has a distinct two peak structure, the total ion energy distribution averaged over the laser channel length very much resembles the Maxwellian energy distribution. In Fig. 17 we show the total number of fast ions, $\left\langle N_{i}\right\rangle$, with energies above the given energy, $\epsilon_{i}$, integrated over the laser channel length: $\left\langle N_{i}\right\rangle=\int d z \int_{\epsilon_{i}}^{\epsilon_{\max }} d \epsilon N_{i}(\epsilon, z)$. One can see an approximately exponential dependence of $\left\langle N_{i}\right\rangle$ on $\epsilon_{i}$ below the energy cutoff at $\sim 550 \mathrm{keV}$. That corresponds to the effective ion temperature $\sim 80 \mathrm{keV}$ (the solid line in Fig. 17) which agrees with the average ponderomotive potential produced by the laser beam. The total number of the fast ions with the energy higher than $100 \mathrm{keV}$ is $\sim 10^{12}$ and with the energy higher than $300 \mathrm{keV}$ is $\sim 3 \times 10^{11}$. These numbers can be significantly increased by using a denser gas target or a larger diameter laser beam. We verified the production of high-energy ions by the direct detection of the significant amount of alpha particles with the energies of $0.1-1 \mathrm{MeV}$, using the nuclear track detector CR-39. The results of these measurements will be published in a following paper.

\section{DISCUSSION AND SUMMARY}

This work demonstrates that interferometric measurements of late time plasma evolution combined with an appropriate theoretical model provide an efficient tool for investigations of a short intense laser pulse interaction with a plasma. In particular, we demonstrate the possibility to retrieve the characteristics of laser channel and fast ions accelerated by the ponderomotive force from the velocity of the late time plasma expansion. We found that the fast ion generation could be a significant channel of the laser energy losses. The average over the channel length total energy of 

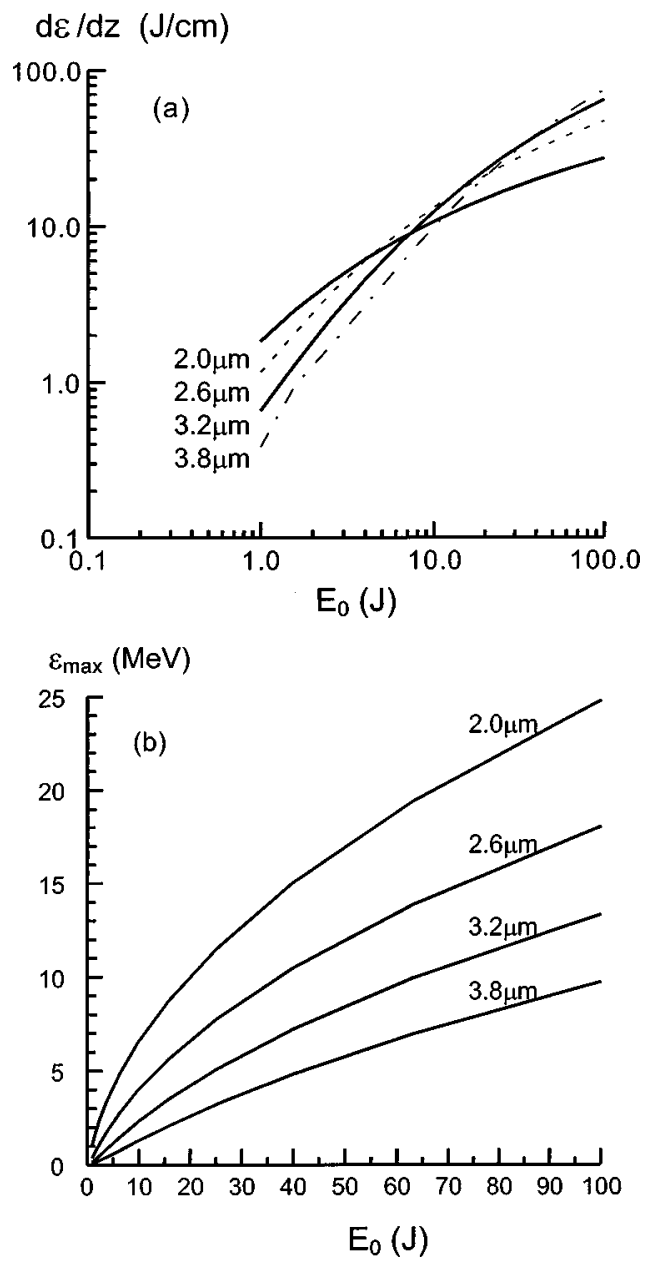

FIG. 18. The dependence of the total energy of fast ions (a) and the maximum ion energy (b) on the laser energy for the laser channel radii $r_{0}=2,2.6,3.2$, and $3.8 \mu \mathrm{m}$, for a laser pulse duration of $400 \mathrm{fs}$ and a He gas density of $5 \times 10^{19} \mathrm{~cm}^{-3}$.

accelerated ions is $\simeq 1.6 \mathrm{~J} / \mathrm{cm}$, that corresponds to a laser pulse dissipation length $L_{a}$ of less than $1 \mathrm{~cm}$. In our experiment, approximately $5 \%$ of the laser energy has been converted into fast ions with an energy of few hundred $\mathrm{keV}$. The efficiency of laser energy conversion into fast ions depends weakly on the laser pulse energy for $E_{0} \gtrsim 1 \mathrm{~J}$, according to Eq. (6). However, the energy conversion will be larger in higher density targets. The estimated energy conversion from Eq. (6) is in reasonable agreement with the model calculations and the experiment. The same is also true for the estimate of maximum ion energy from Eq. (5). For relativistically strong laser pulses, Eqs. (5) and (6) predict the following scalings:

$$
\begin{gathered}
\epsilon_{\max } \approx 77 \frac{Z^{2} \lambda_{0}^{2} E_{0} \tau}{A r_{0}^{4}} \mathrm{MeV}, \\
L_{a} \approx 0.2 \frac{r_{0}^{2} A}{\tau n_{a} Z^{2} \lambda_{0}} \mathrm{~cm},
\end{gathered}
$$

where $E_{0}$ is measured in $\mathrm{J}, \tau$ is in ps, $\lambda_{0}$ and $r_{0}$ are in $\mu \mathrm{m}$, and $n_{a}$ is in $10^{19} \mathrm{~cm}^{-3}$. These relations reasonably (within a factor of 2) estimate the maximum ion energy and laser energy losses for ion acceleration for laser energies in the range of few $\mathbf{J}$, and pulse durations of few tenths of a ps when one can neglect the ion displacement during the acceleration time. For higher laser energies or longer pulse durations, Eqs. (12) overestimate these parameters. Note a sharp dependence of the maximum ion energy on the laser channel radius in Eqs. (5) and (12) and in Fig. 13. Predictions that follow from our model for higher laser pulse energies are presented in Fig. 18 for a constant pulse duration of $400 \mathrm{fs}$. Although both the total energy of fast ions and the maximum ion energy increase with the laser pulse energy, their dependence on the laser channel radius is more complicated. For small laser energies both parameters increase as the laser channel radius decreases. At high laser energies the total ion energy increases with the channel radius while the maximum ion energy decreases. This is another illustration of the fact that most efficient acceleration occurs to ions which have an evacuation time comparable to the laser pulse duration, $r_{0} / v_{i} \sim \tau$.

In summary, we have demonstrated one more application of ultrahigh intensity short laser pulses, a "table-top ion accelerator." The ion acceleration during the process of plasma channel formation constitutes one of the important energy losses for the laser beam in a plasma. Ions in a MeV energy range can be easily generated in experiments with laser pulses of a Joule scale of energy. The laser energy losses for the ion acceleration can be controlled by changing the ambient gas density. The interferometric technique provides an efficient method for the observation of plasma channel formation and ion acceleration by a short intense laser pulse in an underdense gas or plasma.

The interaction of accelerated ions with the ambient gas has been proved to be weakly collisional, and a kinetic model has been developed to describe this interaction. It is shown that the accelerated ions can efficiently excite and ionize the ambient gas atoms, which results in a secondary plasma production long after the end of the laser pulse. This secondary plasma is characterized by a large amount of metastable excited atoms, which might be of interest for some applications. High-energy ions ponderomotively accelerated by intense ultrashort laser pulse in a solid deuteriumtritium target can also produce fusion reactions with a significant yield.

\section{ACKNOWLEDGMENTS}

For constructive suggestions and for supplying us with information on the inelastic cross sections of $\mathrm{He}^{+}$and $\mathrm{He}^{2+}$ ions, we acknowledge Dr. I. Beigman, Dr. D. Uskov, Dr. V. Shevel'ko, Dr. Y. K. Kim, and Dr. M. E. Rudd. Special thanks are due to Dr. R. A. Phanef and K. Flippo for reading the paper, and for useful suggestions. The access to the atomic physics database NIFS is also gratefully acknowledged. G.S.S. is thankful for the support from the Fellowship Program during his visit to the Center for Ultrafast Optical Science. V.Yu.B. and V.T.T. are thankful for the hospitality of the Physics Department of the University of Alberta, Canada where part of this work had been completed. Experimental facilities, as well as A. M. and D. U., were supported by the Divison of Chemical Sciences, Office of Basic Energy Sciences, Office of Energy Research, U.S. Department of Energy. Laser facilities were supported by the National Science Foundation, Center for Ultrafast Optical Science (STC PHY 8920108). V. Yu. B., V. N. N., and V. T. T. were supported by the Russian Foundation for Basic Research. 
[1] M. Tabak, J. Hammer, M. E. Glinsky, W. L. Kruer, S. C. Wilks, J. Woodworth, E. M. Campbell, M. D. Perry, and R. J. Mason, Phys. Plasmas 1, 1626 (1994).

[2] G. A. Mourou, C. P. J. Barty, and D. Perry, Phys. Today 51 (1), 22 (1998).

[3] Advanced Accelerator Concepts: Sixth Annual Conference, edited by P. Schoessow, AIP Conf. Proc. No. 335 (AIP, New York, 1995).

[4] A. B. Borisov, A. V. Borovsky, V. V. Korobkin, A. M. Prokhorov, O. B. Shiryaev, X. M. Shi, T. S. Luk, A. McPherson, J. C. Solem, K. Boyer, and C. K. Rhodes, Phys. Rev. Lett. 68, 2309 (1992).

[5] M. Dunne, T. Afshar-Rad, J. Edwards, A. J. MacKinnon, S. M. Viana, O. Willi, and G. Pert, Phys. Rev. Lett. 72, 1024 (1994).

[6] A. J. Mackinnon, M. Borghesi, A. Iwase, M. W. Jones, G. J. Pert, S. Rae, K. Burnett, and O. Willi, Phys. Rev. Lett. 76, 1473 (1996).

[7] M. Borghesi, A. J. MacKinnon, L. Barringer, R. Gaillard, L. A. Gizzi, C. Meyer, O. Willi, A. Pukhov, and J. Meyer-terVehn, Phys. Rev. Lett. 78, 879 (1997).

[8] D. Umstadter, S. Y. Chen, A. Maksimchuk, G. Mourou, and R. Wagner, Science 273, 472 (1996).

[9] K. Krushelnick, A. Ting, C. I. Moore, H. R. Burris, E. Esarey, P. Sprangle, and M. Baine, Phys. Rev. Lett. 78, 4047 (1997).

[10] V. Malka, E. De Wispelaere, F. Amiranoff, S. Baton, R. Bonadio, C. Coulaud, R. Haroutunian, A. Modena, D. Puissant, C. Stenz, S. Hüller, and M. Casanova, Phys. Rev. Lett. 79, 2979 (1997).

[11] P. Sprangle, E. Esarey, J. Krall, and G. Joyce, Phys. Rev. Lett. 69, 2200 (1992).

[12] S. V. Bulanov, F. Pegoraro, and A. M. Pukhov, Phys. Rev. Lett. 74, 710 (1995)

[13] P. Mora and T. M. Antonsen, Jr., Phys. Rev. E 53, 2068 (1996).

[14] A. Chiron, G. Bonnaud, A. Dulieu, J. L. Miquel, G. Malka, M. Louis-Jacquet, and G. Mainfray, Phys. Plasmas 3, 1373 (1996).

[15] R. Fedosejevs, X. F. Wang, and G. D. Tsakiris, Phys. Rev. E 56, 4615 (1997)

[16] J. Fuchs, G. Malka, J. C. Adam, F. Amiranoff, S. D. Baton, N. Blanchot, A. Héron, G. Laval, J. L. Migel, P. Mora, H. Pépin, and C. Rousseaux, Phys. Rev. Lett. 80, 1658 (1998).

[17] T. Ditmire, E. T. Gumbrell, R. A. Smith, A. Djaoui, and M. H. R. Hutchinson, Phys. Rev. Lett. 80, 720 (1998).

[18] A. J. Mackinon, M. Borghesi, A. Iwase, and O. Willi, Phys. Rev. Lett. 80, 5349 (1998).

[19] R. Annou, V. K. Tripathi, and M. P. Srivastava, Phys. Plasmas
3, 1356 (1996); M. D. Feit, J. C. Garrison, and A. M. Rubenchik, Phys. Rev. E 56, 2394 (1997).

[20] C. A. Coverdale, C. Darrow, C. D. Decker, W. B. Mori, K-C. Tzeng, K. A. Marsh, C. E. Clayton, and C. Joshi, Phys. Rev. Lett. 74, 4659 (1995).

[21] A. Pukhov and J. Meyer-ter-Vehn, Phys. Rev. Lett. 76, 3975 (1996).

[22] G. S. Sarkisov, V. Yu. Bychenkov, V. T. Tikhonchuk, A. Maksimchuk, S. Y. Chen, R. Wagner, G. Mourou, and D. Umstadter, Pis'ma Zh. Eksp. Teor. Fiz. 66, 787 (1997) [JETP Lett. 66, 828 (1997)].

[23] S.-Y. Chen, G. S. Sarkisov, A. Maksimchuk, R. Wagner, and D. Umstadter, Phys. Rev. Lett. 80, 2610 (1998).

[24] P. Maine, D. Strickland, P. Bado, M. Pessot, and G. Mourou, IEEE J. Quantum Electron. 24, 398 (1988); M. D. Perry and G. Mourou, Science 264, 917 (1994).

[25] G. Albrecht, A. Antonetti, and G. Mourou, Opt. Commun. 40, 59 (1981); Y. Beaudoin, C. Y. Chen, J. S. Coe, J. L. Tapié, and G. Mourou, Opt. Lett. 17, 865 (1992).

[26] G. S. Sarkisov, Instrum. Exp. Tech. 39, 727 (1996).

[27] M. V. Ammosov, N. B. Delone, and V. P. Krayinov, Zh. Eksp. Teor. Fiz. 91, 2008 (1986) [Sov. Phys. JETP 64, 1191 (1986)].

[28] S, Augst, D. Strickland, D. D. Meyerhofer, S. L. Chin, and J. H. Eberly, Phys. Rev. Lett. 63, 2212 (1989).

[29] http://www.scioncorp.com

[30] Y. B. Zel'dovich and Y. P. Raizer, in Physics of Shock Waves and High-Temperature Hydrodynamic Phenomena, edited by W. D. Hayes and R. F. Probstein (Academic, New York, 1966).

[31] J. F. Luciani, P. Mora, and J. Virmont, Phys. Rev. Lett. 51, 1664 (1983); J. R. Albritton, E. A. Williams, I. B. Bernstein, and K. P. Swartz, ibid. 57, 1887 (1986); V. Yu. Bychenkov, W. Rozmus, V. T. Tikhonchuk, and A. V. Brantov, ibid. 75, 4405 (1995).

[32] M. B. Shah, D. S. Elliott, P. McCallion, and H. B. Gilbody, J. Phys. B 21, 2751 (1988); R. G. Montague, M. F. A. Harrision, and A. C. H. Smith, ibid. 17, 3295 (1984).

[33] M. E. Rudd, T. V. Goffe, and A. Itoh, Phys. Rev. A 32, 2128 (1985); M. B. Shah and H. B. Gilbody, J. Phys. B 18, 899 (1985).

[34] R. K. Janev, Phys. Rev. A 53, 219 (1996).

[35] N. H. Burnett and G. D. Enright, IEEE J. Quantum Electron. 26, 1797 (1990).

[36] C. K. Birdsall and A. B. Langdon, Plasma Physics via Computer Simulation (McGraw-Hill, New York, 1985).

[37] H. R. Griem, Plasma Spectroscopy (McGraw-Hill, New York, 1964). 